\title{
Quand les Apatrides et les Infidèles contestent : territoires, conflits, innovations
}

When the Stateless and the Infidels Protest: Territories, Conflicts, Innovations

\section{Pinar Selek}

\section{(2) OpenEdition}

12 Journals

\section{Édition électronique}

URL : http://journals.openedition.org/conflits/19321

DOI : $10.4000 /$ conflits. 19321

ISSN : $1777-5345$

Éditeur :

CCLS - Centre d'études sur les conflits lilberté et sécurité, L'Harmattan

\section{Édition imprimée}

Date de publication : 8 août 2016

Pagination : 165-187

ISBN : 978-2-343-10075-3

ISSN : 1157-996X

Référence électronique

Pinar Selek, "Quand les Apatrides et les Infidèles contestent : territoires, conflits, innovations », Cultures \& Conflits [En ligne], 102 | été 2016, mis en ligne le 08 août 2018, consulté le 30 mars 2021. URL : http://journals.openedition.org/conflits/19321 ; DOI : https://doi.org/10.4000/conflits. 19321 


\section{Quand les Apatrides et les Infidèles contestent : territoires, conflits, innovations}

\section{Pinar SELEK}

Pinar Selek est sociologue, docteure en science politique, docteure honoris causa de l'ENS de Lyon et ATER en science politique à l'Université de Nice Sophia Antipolis. Elle est membre associée de SAGE (UMR 7363, Université de Strasbourg) et de l'URMIS (UMR 8245). Dernière publication : "Comment le féminisme peut contribuer à la création de la politique? ", in Anthologie politique de Turquie, Vienne, Vienna Institute for International Dialogue and Cooperation, 2016.

T e génocide des Arméniens de 1915 n’a été médiatisé que tardivement, au Univeau international, en 2015, à l'occasion de son centenaire. Les chercheurs de plusieurs pays avaient déjà mis en évidence ses causes sociales et politiques. Nous savons ainsi que les Jeunes Turcs avaient pour projet de transformer l'État religieux en un État-nation et de construire une économie nationale. À l'ombre de la Première Guerre mondiale, ils mirent à exécution, de mai à juillet 1915, une opération d'extermination de la communauté arménienne ${ }^{1}$. Sur les 2,5 millions d'Arméniens que comptait l'Empire ottoman en 1914, les deux tiers - 1,5 millions - ont péri et environ 800000 survivants, laissant derrière eux leurs biens qui avaient été confisqués, se sont dispersés sur les cinq continents, après avoir transité par plusieurs destinations d'infortune ${ }^{2}$. Suite à leur déterritorialisation forcée, ces survivants forment la diaspora des «Apatrides » structurée à travers des organisations politiques. Quant aux Arméniens demeurés en Turquie, ils sont présentés comme «Infidèles » dans les livres de l'Éducation nationale. «La trahison des Arméniens » est une

1. Voir notamment : Akçam T., From Empire to Republic: Turkish Nationalism and the Armenian Genocide, London, Zed Books, 2004 ; Dadrian V., Histoire du Génocide arménien, Paris, Stock, 1996 ; Kevorkian R., Le génocide des Arméniens, Paris, Odile Jacob, 2006 ; Ternon Y., Les Arméniens et l'histoire d'un génocide, Paris, Seuil. 1977 ; Ternon Y., Kevorkian R., Mémorial du Génocide des Arméniens, Paris, Seuil, 2015.

2. Mouradian C., Kunth A., Les Arméniens en France, Toulouse, Éditions de l'attribut, 2010. 
partie constituante du récit imaginaire du nationalisme turc ${ }^{3}$. En dehors de ceux et celles qui sont convertis à l'Islam, environ 60000 de ces «Infidèles » ayant un statut de minorité religieuse, se dissimulent à l'ombre de l'Église grégorienne, sans avoir de relations importantes avec la diaspora 4. Et cela, jusqu'aux années 2000 .

Si les travaux académiques sur la diaspora arménienne, ses configurations sociales et culturelles, ses représentations et ses comportements politiques apparaissent à partir des années 1990, la recherche universitaire sur les mobilisations politiques des Arméniens n'en est encore qu'à ses débuts ${ }^{5}$. Cet article est issu d'une recherche sur les mobilisations politiques de la diaspora arménienne en France qui est leur premier foyer européen ${ }^{6}$ et qui rassemble une population dix fois plus nombreuse qu'en Turquie. Notre étude a été menée, entre 2014 et 2016, dans plusieurs villes de France ainsi qu'en Belgique, en Italie, en Suisse, en Turquie et en Arménie, afin de saisir les effets et les liens transnationaux 7 . Elle englobe les différentes étapes des trajectoires des mouvements politiques arméniens pour examiner les multiples facteurs qui contribuent à façonner ces mobilisations. Le présent article se centre sur des évolutions de la structure de la diaspora arménienne depuis ses origines jusqu'à aujourd'hui, en fonction des évolutions de la scène internationale (au niveau des États) et transnationale (au niveau des mouvements sociaux). Pour comprendre la situation actuelle, ses mutations récentes et à venir, il s'agit de montrer les liens entre les trajectoires politiques et territoriales des mobilisations, autrement dit de voir comment la diaspora politique s'est construite historiquement et a déjà profondément évolué en fonction des opportunités et des restrictions imposées par les relations internationales. Nous montrerons comment la politique "par le haut », celle des États et des relations internationales,

3. Selek P., Parce qu'ils sont arméniens, Paris, Liana Levi, 2015.

4. Karakaşlı K., Kentel F., Özdoğal G., Üstel F., Les Arméniens en Turquie, Istanbul, Presses universitaire Bilgi, 2009, p. 25.

5. Les travaux de Gaidz Minassian sont remarquables sur ce sujet: Guerre et terrorisme arméniens, Paris, PUF, 2002 ; Arméniens - Le temps de la délivrance, Paris, CNRS Histoire, 2015.

6. Aujourd'hui, sur une population arménienne mondiale estimée à 11 millions d'individus, moins de trois millions résident en Arménie, 1,5 million aux États-Unis, 60000 en Turquie, plus de 600000 en France. Voir : Ekovich S., « La géosociologie de la diaspora arménienne », Géostratégiques, $\mathrm{n}^{\circ}$ 12, «La géosociologie de l'Asie centrale du Sud », avril 2006, pp. 193210.

7. Il s'agit d'un travail de terrain effectué au sein de Triangle (UMR 5206) entre 2014 et 2015 et de SAGE (UMR 7363), entre 2015 et 2016. Durant cette enquête conduite au travers d'observations participantes, nous avons réuni un nombre important de sources de première main, à la fois écrites (archives, textes d'accompagnement de formations internes, notes manuscrites, publications, affiches, slogans, programmes, communiqués, statuts, documents de revendication, déclarations de presse, brochures, manifestes, revues, sites web) et orales (retranscription des entretiens et de discussions internes lors de réunions de ces organisations). Les 42 entretiens avec les militants des mouvements de la diaspora (31) et de la Turquie (11) forment un échantillon qui représente les différentes catégories d'âge, de sexe, de classe, de responsabilités, de tendances, d'expériences dans ces mouvements. Nous avons opté pour des entretiens semi-structurés, réalisés de manière souple et interactive, afin de permettre aux personnes interviewées d'exprimer plus librement leurs expériences individuelles. Ces conversations libres ont néanmoins respecté une liste de sujets à traiter préalablement établie. 
contribue à définir la " politique par le bas », celle des mouvements, tout en étant influencée par cette dernière. Par une analyse longitudinale, nous expliquerons les derniers développements de la diaspora et ses relations avec le mouvement arménien turc actuel. Nous verrons dans un premier temps comment la structuration politique de la diaspora donne lieu à des lignes de fracture avec les Arméniens de Turquie, tout en favorisant les opportunités de contestation dans ce pays. En dernier lieu, nous envisagerons les bases sur lesquelles les mobilisations favorisent le développement de réseaux transnationaux qui dépassent les frontières nationales ainsi qu’organisationnelles.

\section{Du silence à la contestation : la structuration politique de la diaspora arménienne}

Si une partie de la population d'origine arménienne en France est issue de la première vague d'émigration qui suivit les massacres de 1894-1896, la majeure partie migre entre 1925 et1938, en suivant des itinéraires chaotiques ${ }^{8}$. Ce n'est pas par hasard que la France est le premier foyer européen des Arméniens. Plusieurs travaux - notamment l'étude de Vincent Duclert - ont relevé en France la visibilité importante de la contestation contre les grands massacres de 1894-1896 dans l'Empire ottoman 9. Après le génocide de 1915, cette sensibilité s'élargit aux Alliés qui, par l'intermédiaire d'une déclaration commune le 24 mai 1915, proclament qu'ils tiendront les membres du gouvernement ottoman pour responsables de crimes contre l'bumanité et la civilisation 10. Pourtant, même si cette déclaration et les "procès des Unionistes » (1919-1920) conduits à Istanbul, donnent naissance à un nouveau cadre pour un droit pénal international en introduisant le concept de « crime contre l'humanité », ils restent sans suite ${ }^{11}$. En 1923, les Alliés signent avec le gouvernement kémaliste le Traité de Lausanne qui ne fait aucune référence aux crimes. La Turquie, voisine de l'URSS, devient un enjeu stratégique majeur en Méditerranée et tisse des liens économiques et politiques avec les grandes forces occidentales ${ }^{12}$.

Claire Mouradian et Annouche Kunth expliquent que dans cette période, l'aide humanitaire se substitue à l'aide politique ${ }^{13}$. Celle-ci n'empêche pourtant pas la violence hostile que les Arméniens subissent dès leur arrivée en

8. Poinsot M., « L’arménien occidental, cent ans de diaspora », Hommes et migrations, $\mathrm{n}^{\circ} 1310$, 2015, URL : http://hommesmigrations.revues.org/3194 (consulté le 16 mai 2016); Mouraidian C., Kunth A., op. cit.

9. Duclert V., La France face au génocide des Arméniens, Paris, Fayard, 2015 ; Jaurès J., Il faut sauver les Arméniens, Paris, Mille et Une Nuit, 2015.

10. Rapport de la Commission des Affaires étrangères sur la proposition de loi de M. Didier Migaud relative à la reconnaissance du génocide arménien de 1915, par René Rouquet, Assemblée nationale, $\mathrm{n}^{\circ}$ 925, 26 mai 1998, p. 13.

11. Dadrian V., Akçam T., Jugement à Istanbul, La Tour d'Aigues, Éditions de l'Aube, 2015.

12. Gilquin M., D'Antioche au Hatay. L'Histoire oubliée du Sandjak d'Alexandrette, Paris, l'Harmattan, 2000.

13. Mouradian C., Kunth A., op. cit., p. 17. 
France par bateau au port international de Marseille. La lettre du Maire de Marseille de l'époque, publiée en octobre 1923, dans le journal Le Petit Provençal, n'est qu'un exemple :

\begin{abstract}
«Depuis quelques temps se produit vers la France, un redoutable courant d'immigration des Arméniens. [...] la peste se dirige vers nous, $[\ldots]$ ces immigrants, dénués de tout, réfractaires aux mœurs occidentales, rebelles à toute mesure d'hygiène, immobilisés dans leur indolence résignée, passive, ancestrale. [...] La population de Marseille réclame du gouvernement qu'il interdise l'entrée des ports français à ces lamentables troupeaux humains, gros danger public pour le pays... ${ }^{14}$ ».
\end{abstract}

Cette hostilité est conforme aux raisons de leur accueil en France. À l'époque, l'aide humanitaire mobilise les pays qui sont en quête de maind'œuvre : les survivants obtiennent le statut d'Apatrides élaboré, en 1922, par le Haut-commissariat pour les Réfugiés de la Société des Nations via l'Office International Nansen pour les Réfugiés, qui leur permet de circuler, de travailler et d'être reçus par les pays qui ont besoin de force de travail ${ }^{15}$. D'après les estimations du Bureau International du Travail 16, c'est le cas de la France qui « exerce dès le début des années 1920 son mandat sur la Syrie et le Liban, et les Arméniens sont recrutés sur place par des entreprises françaises 17 ». Jusqu'aux années 1930, 66000 Apatrides, titulaires d'un passeport dit « passeport Nansen ", avec l'indication «sans retour possible », tombent dans une «brusque prolétarisation » 18 .

L'hostilité favorise leur précarisation, leur fragilité administrative, et les contraints à la discrétion. Bien que la criminalisation des migrations soit un concept conçu récemment pour aborder la complicité entre l'État et les employeurs à la fin du Xx ${ }^{\mathrm{e}}$ siècle ${ }^{19}$, il permet d'appréhender la situation de ces non-citoyens du début du siècle. N'ayant pas recours à la protection du travailleur, ils acceptent les lois de l'offre : «en manque de l'intérêt de l'État, les entreprises envoient des agents recruteurs dans les camps de réfugiés 20 ».

14. Marchand L, Perrier G, La Turquie et le fantôme arménien, Marseille, Actes Sud, 2013, pp. 28-29.

15. Mouradian C., Kunth A., op. cit., p. 18.

16. Ter Minassian A., "Les Arméniens à Paris, 1839-1939 ", in Histoires croisées, Marseille, Parenthèses, 1997, p. 62. Les chiffres varient selon les sources et leur fiabilité est douteuse.

17. Ritter L., La longue marche des Arméniens : histoire et devenir d'une diaspora, Paris, Robert Laffont, 2007, p. 16.

18. Ter Minassian A., "Les Arméniens en France ", Les Temps modernes, numéro spécial, 1988.

19. Balibar E., Chemiller-Gendreau M., Costa-Lascoux J., Terray E. (dir), Sans-papiers, l'archaisme fatal, Paris, La Découverte, 1999 ; Waldinger R.D., " The making of an immigrant niche », International Migration Review, vol. 8, n 1, 1994, pp. 3-30; Pallida S., "La criminalisation des migrants ", Actes de la recherche en sciences sociales, $\mathrm{n}^{\circ} 129$, septembre 1999, pp. 39-49; Sayad A., La Double Absence. Des illusions de l'émigré aux souffrances de l'immigré, Paris, Seuil, 1999.

20. Mouradian C., Kunth A., op. cit., p. 21. 
Après un séjour dans ces camps à Marseille, ils s'implantent, par l'intermédiaire d'agents recruteurs, dans la périphérie de la cité phocéenne, en province, dans les régions Rhône-Alpes et Ile-de-France, où ils créent plusieurs petites colonies arméniennes. La destruction physique, le déracinement de la population et le changement de position des Alliés face à la Turquie, convergent pour entraver la situation des exilés, mais aussi pour encourager le regroupement. Les « espaces de l'entre soi » dont parle Martine Hovanessian permettent à cette population traumatisée de se reconstruire sur un mode communautaire 21. Elle explique en quoi, pour les réfugiés, la construction d'une mémoire collective et d'un futur national mobilisent des ressources puisées dans la rencontre avec les codes normatifs des sociétés d'accueil 22. Dans les témoignages recueillis durant notre enquête, le constat commun des militants arméniens de toutes les générations, des diverses organisations, est que cette période est caractérisée par le silence «mais également par la volonté de conserver la mémoire 23 ». Les commémorations du 24 avril ${ }^{24}$, date considérée comme le début du génocide de 1915, l'attestent. Ces événements se déroulent dans un cadre religieux. Les apatrides conservent, dans l'ombre de l’Église, leur mémoire de la violence et du « pays perdu ». Un Marseillais d'origine arménienne qui avait vingt ans à la fin des années 1950, précise que l’Église était le seul refuge, jusqu'aux années 1970, pour la communauté. «Parce qu'on ne nous permettait pas plus. À plusieurs reprises, la police est intervenue au sein même de nos églises pour interdire les commémorations. Pas le droit de parler du génocide de 191525 ». Les recherches de V. Duclert, qui montrent l'ampleur de la répression politique des gouvernements français autour de cette question, nous aident à comprendre pourquoi la mémoire devient un enjeu et est souvent présentée comme ce qui doit être mis à l'abri 26. «Rendus amers par l'indifférence du monde à leurs malheurs, les Arméniens de la diaspora gardent pour eux leurs frustrations, leur traumatisme, et même leurs talents créatifs » indique Hratch Dasnabedian 27. Dans les témoignages recueillis durant notre recherche ${ }^{28}$, il y a une récurrence des termes " amertume » ou «frustration ». «Pleurer ensemble » devient ainsi un élément constitutif de l'identité arménienne, en diaspora. «Les rassemblements du 24 avril, organisés par l'Église étaient épouvantables. Les gens pleuraient. C'était triste. Dans mon enfance, tous les événements communautaires, même les

21. Hovanessian M., Le Lien communautaire, Paris, Armand Colin, 1992.

22. Hovanessian M., « Diasporas et identités collectives », Hommes et migrations, $\mathrm{n}^{\circ} 1265$, janvier-février 2007, pp. 8-21 ; Hovanessian M., « Le religieux et la reconnaissance. Formes politiques et symboliques au sein de la diaspora arménienne», Les Annales de la recherche urbaine, $\mathrm{n}^{\circ}$ 96, octobre 2004, pp. 125-134.

23. Entretien avec Séta Papazian, 9 octobre 2014, Paris.

24. Le 24 avril 1915, commence l'opération contre 600 notables arméniens qui seront assassinés sur ordre du gouvernement de l'Empire ottoman.

25. Entretien avec T.K., 8 mai 2015, Marseille.

26. Duclert V., op. cit.

27. Dasnabedian H., Histoire de la FRA Dachnaktsoutioun 1890-1924, Oemme, Collection Muhrak, 1988, p. 20.

28. Sur les 31 témoignages des militants de diaspora, 28 ont utilisé ces termes. 
mariages, finissaient par des séances de pleurs » évoque une parisienne d'origine arménienne de 65 ans. C'est autour de cette identité, faite de déracinement et d'horreur, que la diaspora arménienne se structure progressivement.

La rupture avec un territoire structurant est souvent évoquée dans les études des diasporas. Utilisé d'abord comme nom propre désignant la dispersion du peuple juif, le terme de « diaspora » est ensuite appliqué - sans majuscule - à toute forme de dispersion d'importance des peuples ou nations, pourvu que cette dispersion ne soit pas éphémère ${ }^{29}$. Les diasporas sont traditionnellement définies comme des groupes sociaux subissant, à leur origine, des évictions forcées et une installation dans deux ou plusieurs lieux différents hors de leur foyer ancestral, tout en ayant conservé des marqueurs communautaires. L'évolution du terme, au cours des dix dernières années, du fait d'un nombre croissant de travaux s'inscrivant dans les transnational studies ou les diaspora studies qui tendent à couvrir une large réalité de groupes migrants, comme expatriés, exilés, réfugiés, immigrés, rend difficile une définition commune du terme diaspora, source de nombreux conflits au sein de la communauté scientifique. Pour répondre à l'inconfort actuel de l'usage du terme, Stéphane Dufoix l'historicise et le redéfinit depuis l'origine : la traduction grecque du bannissement, de la terreur, mais aussi de la préservation et de la semence ${ }^{30}$. Il ne suffit pas de dépasser l'effondrement de plus en plus marqué de la distinction entre diaspora et dispersion pour « considérer le phénomène de la diaspora dans des contextes divers et des rôles nouveaux » comme le dit Khachig Tölölyan ${ }^{31}$. Sur ce point, S. Dufoix met en avant l'avantage de divers usages du terme qui permettent d'articuler les contraires, de lire les réalités d'un monde en mouvement et de les rendre plus intelligibles. Pour lire la réalité du cas arménien, la définition de M. Hovanessian nous semble plus pertinente : une nouvelle catégorie de l'altérité, conçue comme une construction dynamique, un espace de fiction actif et non comme une catégorie figée ${ }^{32}$. En évaluant la pertinence des notions telles que la stratégie identitaire, la mémoire collective, l'héritage culturel, en les mettant en relation avec le sentiment d'appartenance et l'imaginaire national, $M$. Hovanessian indique que ces notions interviennent pour rendre compte des identités singulières qui, dans le cas de la diaspora arménienne, s'élaborent à partir d'une rupture violente et radicale : celle du génocide de 1915.

Les différents visages de cette diaspora selon les pays d'implantation et la géographie mouvante sur laquelle elle se construit, peuvent nous permettre, comme le propose Laurence Ritter, d'aborder au pluriel le phénomène dias-

29. Médam A., "Diaspora/Diasporas. Archétype et typologie », Revue européenne des migrations internationales, vol. 9, $\mathrm{n}^{\circ} 1,1993$, pp. 59-66.

30. Dufoix S., Les diasporas, Paris, PUF, 2003.

31. Tölölyan K., "The Contemporary Discourse of Diaspora», Relations Internationales, $\mathrm{n}^{\circ} 141$, 2010, p. 25.

32. Hovanessian M., art. cité, 2007, p. 9. 
porique, c'est-à-dire, de parler de plusieurs diasporas arméniennes ${ }^{33}$. Notre enquête a vérifié ce constat, comme celui de C. Mouradian et A. Kunth qui disaient que la diaspora arménienne " apparaît très composite, traversée de multiples clivages et en constante reconfiguration 34 ». L. Ritter le précise, « elle est tout sauf un bloc monolithique » car « elle est marquée par sa propre histoire, sa propre temporalité, ses propres modes d'évolution, à distinguer suivant les pays d'implantation 35 ». Dans son livre elle montre que c'est l'historique des implantations diasporiques qui influe sur leur visage d'aujourd'hui. Nous pouvons aller plus loin : même dans un seul pays, on observe une grande diversité selon les régions et les villes et il est donc possible de mettre en avant la réalité effectivement composite des communautés. Mais qu'estce qui «fait » communauté ? La distance vaut-elle détachement ou attachement ? À partir de ces questions, S. Dufoix avance son analyse et relève deux traits caractéristiques de cette catégorie : «multipolarisation de la migration d'un même groupe entre différents pays et interpolarité des relations 36 ». Il soutient que la conception décentrée de diaspora est, elle, à la source d'un recentrement de celle-ci et d'une redéfinition de l'espace dans lequel les proximités et les sociabilités se jouent. Dans ce sens, nous utiliserons le terme au singulier car il est également fort apparent qu'en dépit de cette hétérogénéité, il est question d'une identité transnationale, composite mais traversée par la même histoire politique, reconstruite au fil du temps. Bien que dans le domaine des recherches empiriques, tout comme dans celui de la réflexion théorique, il existe diverses définitions de transnationalisme ou d'espace transnational ${ }^{37}$, son sens général qui s'intéresse aux échanges, aux relations et aux pratiques transfrontalières transcendant le cadre national en tant que principal point de repère pour l'exercice d'une activité ou l'affirmation d'une identité, nous servira pour préciser que cette diaspora ne peut pas être distinguée uniquement selon les pays d'implantation, mais aussi à partir d'une construction commune qui dépasse les frontières de ces pays. Nous rejoignons l'analyse de M. Hovanessian qui explique comment l'exil et la violence de l'exclusion contribuent à ranimer une histoire ponctuée de bouleversements politiques et comment la diaspora arménienne, même dans différents pays, se caractérise par l'abandon sur la scène internationale de la question arménienne. Quels sont alors les autres éléments constituants de cette formation ? Pour avancer,

33. Ritter L., op. cit.

34. Mouradian C., Kunth A., op. cit., p. 9.

35. Ritter L., "L’identité arménienne et turque aujourd'hui », Repairfuture, 2014, URL : http://repairfuture.net/index.php/fr/l-identite-point-de-vue-de-la-diaspora-armenienne/lidentite-armenienne-en-diaspora-entre-modernite-et-preservation (consulté le 5 février 2015).

36. Dufoix S., op. cit., 2003 ; Dufoix S., La dispersion. Une histoire des usages du mot diaspora, Paris, Éditions Amsterdam, 2011.

37. Glick-Schiller N., Levitt P., « Conceptualizing simultaneity. A Transnational Social Field Perspective on Society", in Portes A., DeWind J. (dir.), Rethinking Migration. New Theoretical and Empirical Perspectives, New York, Berghahn Books, 2007, pp. 181-218; Morokvasic M., «La mobilité transnationale comme ressource : le cas des migrants de l'Europe de l'Est », Cultures E Conflits, n³3-34, 1999, pp. 105-122. 
nous reprenons la question qu'elle pose : pourquoi certains groupes revendiquent-ils un statut diasporique à tel moment de leur histoire 38 ? Précisons : Pourquoi cette revendication émerge-t-elle à la fin des années 1960, au sein de la communauté arménienne qui s'est reconstruite en dehors de la Turquie et d'Arménie ? Aborder les enjeux de l'auto-désignation peut nous aider à mieux comprendre l'interdépendance des mobilisations sociales et politiques.

L'organisation communautaire diasporique ne se réalise pas uniquement autour de l'Église, mais aussi autour des trois partis politiques arméniens : Dachnak (1890), Hentchak (1897) et Ramgavar (1885-1908) qui, avant le déracinement, sont implantés dans les territoires de l'Empire ottoman, de l'Empire russe, de l'Iran et de l'Europe. Ces partis avaient pris part à trois révolutions : la révolution russe en 1905, la révolution des libéraux perses contre le Chah, en 1906-1908, et la révolution ottomane en 1908. Ils appartenaient à un mouvement multilatéral qui réunissait également plusieurs groupes indépendants. Ce mouvement se nourrissait des débats déchainés par la circulation des idées nationalistes, marxistes et populistes, qu'en retour il servait à diffuser entre plusieurs territoires, " par-delà les frontières ». L'élimination presque totale de la population arménienne en Anatolie, implique inévitablement l'éradication de ces organisations politiques de leurs territoires d'origine et leur reconstruction dans plusieurs territoires d'adoption. Leur caractère multilatéral, depuis plus d'un siècle, facilite leur adaptation aux nouvelles conditions qui s'offrent à elles. Elles s'appuient sur la mémoire comme ressource politique. La notion de ressource dans l'analyse de l'action collective nous permet de désigner les stocks hétérogènes d'avoirs, de savoirs, d'images et de pratiques qui constituent les munitions de tactiques et stratégies de lutte ${ }^{39}$. Les groupes politiques se développent au sein des liens sociaux et économiques qui leur fournissent les diverses ressources nécessaires au lancement et au maintien de l'action collective. Dans la poursuite de leurs buts, les mouvements protestataires assemblent et investissent leurs ressources, indique Anthony Oberschall 40. Nous distinguons le même processus dans l'histoire des groupes politiques arméniens. Suite à la déterritorialisation absolue de cette population, commence une période de survie et de reconstruction sociale dans plusieurs pays. C'est une étape de préparation, une période de capitalisation des ressources.

En se reconstituant, ces organisations contribuent à la construction même de la communauté diasporique dont elles font partie ${ }^{41}$, par exemple elles favo-

38. Hovanessian M., art. cité, 2007, p. 12.

39. Carthy J. D., Zald M. N., "Ressource Mobilization and Social Movements: A Partial Theory ", American Journal of Sociology, vol. 82, n 6, 1977, p. 1217 ; Neveu E., " Répertoires d'action des mobilisations ", in Cohen A., Lacroix B., Riutort P. (dir), Nouveau manuel de science politique, Paris, La Découverte, 2009, p. 497.

40. Oberschall A., Social Conflicts and Social Movements, New Jersey, Prentice-Hall Inc, 1973, p. 28.

41. Ritter L., La longue marche des Arméniens : histoire et devenir d'une diaspora, op. cit., p. 133. 
risent le maintien mais également la réélaboration de la mémoire qui n'est désormais plus réduite au seul crime car elle va s'enrichir des récits de résistances. En France, nous avons vu les photos de Soghomon Tehlirian dans plusieurs espaces arméniens et observé qu'il est l'un des plus importants symboles de cette mémoire. Le 24 avril 2016, les organisations arméniennes annoncent la construction d'une statue, à Marseille, en hommage à Tehlirian qui, le 15 mars 1921, avait assassiné Talaat Pacha 42 à Berlin, dans le cadre de l'Opération Némésis menée par le parti Dachnak (1920-1922), et cela afin d'exécuter les sentences des tribunaux d'Istanbul, prononcées par contumace. Ces procès s'achèvent par la condamnation à mort des responsables du génocide qui avaient fui notamment en Allemagne. En 1921, arrêté par la police allemande, Tehlirian était acquitté, lors de son procès, après une plaidoirie historique qui demandait justice pour les victimes. H. Arendt relève dans ses écrits son insistance lors du procès à être jugé pour «montrer au monde, grâce à l'exposition judiciaire, que des crimes restaient impunis 43 ». Cette action reste un des évènements constitutifs pour la diaspora arménienne.

La visibilité des photos d'une autre personne a attiré notre attention durant notre recherche : celles de Missak Manouchian ${ }^{44}$, symbole de la participation des Arméniens à la Résistance en France, pendant la Seconde Guerre mondiale. Nous avons pu évaluer facilement le niveau de cette participation par la quantité de noms arméniens gravés sur les monuments dédiés aux "Morts pour la France », dans plusieurs villes de l'hexagone. Même si plusieurs Arméniens pensent que ces « étrangers » bien que résistants, n’ont pas été protégés par les organisations de résistance et ont été exposés à la répression nazie, nous estimons que cette implication renforce leurs liens en dehors de la communauté. En tout cas, ces Apatrides formaient, avant le génocide, la classe des artisans, des producteurs, des commerçants, dans l'Empire ottoman. Malgré la précarité qui leur a été imposée, ce capital éducatif et professionnel facilite leur intégration économique puis sociale, accélérée par leur engagement dans la Resistance durant la Seconde Guerre mondiale. En conséquence, la deuxième génération réunit des fonctionnaires, des médecins, des architectes, des banquiers, des industriels, des universitaires, intégrés à la société française, ce qui renforce les ressources des organisations politiques arméniennes, construites sur la même base sociale multipolaire. Cela va de pair avec la conservation, la politisation, la reconstruction et la mobilisation de la mémoire. Même si les clivages sont forts entre Hentchak qui soutient l'Arménie soviétique, Ramgavar qui essaie de s'arranger avec cette réalité et Dachnak qui est antisoviétique, ces partis politiques s'allient autour des buts prioritaires de l'époque : conserver la mémoire, créer des structures pour construire une identité nationale arménienne ${ }^{45}$ qui devient, en diaspora, de

42. Il est considéré comme le principal organisateur du génocide de 1915.

43. Arendt H., Rapport sur la banalité du mal, Paris, Gallimard, 1991.

44. Missak Manouchian (1906), résistant communiste fusillé à 37 ans, le 21 février 1944. 
plus en plus imaginée, au sens où l'entend Benedict Anderson ${ }^{46}$. Ils mettent ainsi en place des associations, fondations, réseaux de solidarités ou espaces culturels. Il s'agit de construire des ressources permanentes afin de mener une lutte à long terme ${ }^{47}$. Le point important est l'articulation des ressources économiques, sociales et politiques, ainsi que la transformation des résistances quotidiennes en mobilisations collectives 48 .

Cette structuration fait naître une communauté qui n'entre pas dans les catégorisations rapides. N. Glick-Schiller et L. Basch distinguent les différents types de migrants : les émigrés sont tournés vers le pays d'arrivée tandis que les transmigrants construisent un champ social, à travers leurs activités sociales, économiques et politiques qui déborde des frontières nationales et qui s'inscrit simultanément dans le pays de départ et dans celui d'arrivée : ils sont présents et font des ponts entre ces deux sociétés ${ }^{49}$. Le cas de la diaspora arménienne permet de voir que la démarcation entre ces notions n'est pas statique. Cette diaspora est constituée à l'origine des rescapés du génocide de 1915 qui n’a jamais été reconnu par le pays de départ. Il n’y a donc pas de relations avec ce dernier, ni avec les Arméniens restés en Turquie. Durant des décennies, les Apatrides, chassés de leur "patrie lointaine ", tournés vers les pays d'arrivée, s'y intègrent aux niveaux social, économique et politique. Pourtant s'il s'agit d'une rupture avec le territoire qu'ils ont fui, ils le font vivre dans leurs nouveaux espaces : tout en s'intégrant aux sociétés d'accueil, ils gardent une identité politique apatride et une identité culturelle étrangère. L'émergence des organisations politiques favorise le maintien de la mémoire $\mathrm{du}$ « pays perdu » et des espaces culturels arméniens dans plusieurs pays qui créent des ponts entre les Arméniens intégrés à ces pays. Le témoignage d'un ex-militant de la JAF (Jeunesse Arménienne de France-1945), affirme le rôle important des organisations politiques dans la construction dynamique de la communauté : «Grâce aux scouts de la jeunesse arménienne, j’ai connu plusieurs arméniens du Canada, du Liban, de Suisse... même d'Afrique 50 ».Né en 1947 dans une famille de rescapés, cet instituteur retraité, qui a toujours vécu à Marseille, raconte que les réunions politiques auxquelles il a participé dans sa jeunesse ont suscité des liens qui lui servent toujours pour des voyages touristiques, échanges culturels, réseaux d'information, etc. Ce témoignage montre comment la distance qui sépare les communautés dans les différents

45. Kouyoumdjian J., "Diaspora en France - Les partis politiques ", France-Arménie, n 73, 1988.

46. Anderson B., Imagined Communities, Londres, Verso, 2002.

47. Carthy J. D., Zald M. N., art. cité, p. 1217.

48. Voir : Fillieule $\mathrm{O}$, « De l'objet de la définition à la définition de l'objet. De quoi traite finalement la sociologie des mouvements sociaux ? ", Politique et Sociétés, vol. 28, n 1, 2009, pp. 15-36.

49. Glick-Schiller N., Levitt P., op. cit. ; Shiller N, Basch L., Blanc-Szanton C, «From Immigration to Transmigrant : Theorizing Transnational Migration », Anthropoligical Quarterly, ${ }^{\circ} 68,1995$, pp. 48-63.

50. Entretien avec M.D., 7-8 janvier, Nice. 
pays est ainsi traversée par le réseau militant qui s'affranchit des contraintes géographiques tout en fournissant des liens de sociabilité. S’il ne s'agit pas de relations avec le pays de départ, ce réseau qui lie les individus de différents mondes sociaux autour de valeurs ou d'intérêts communs façonne une identité multipolaire qui est une identité de réseau, une identité transnationale. Cette configuration rappelle la notion de " communauté transnationale ", conceptualisée par A. Portes même s'il désigne les réseaux d'entrepreneurs qui «traversent les frontières nationales et qui ne se situent véritablement "ni ici ni là” mais ici et là en même temps 51 ». Dans le cas des Arméniens, ce sont les organisations politiques qui créent les réseaux de relations denses qui traversent les frontières politiques. Sans être pour autant un pont entre les pays de départ et les pays d'arrivée, elles construisent un pays perdu des Apatrides sur un espace qui englobe plusieurs pays.

Une autre ressource, cette fois-ci juridique, se construit dans le sillage de la Seconde Guerre mondiale et de l'extermination des juifs d'Europe. Rafael Lemkin (1900-1959), juriste polonais, en s'inspirant des cas des Chrétiens de Rome, des Arméniens de l'Empire ottoman et des Juifs pendant la Seconde Guerre mondiale, crée le terme de génocide, en 1944. Le vocable existe juridiquement à partir de 1948, date de la Déclaration Universelle des Droits de l'Homme et de la Convention pour la prévention et la répression du crime de génocide. Même si elle visait uniquement la destruction des Juifs d'Europe, cette déclaration fournit aux organisations arméniennes un cadre juridique pour la formulation de la revendication de la reconnaissance du génocide de 1915. "Ajoutant des arguments juridiques aux aspects politiques de la cause arménienne 52 », elles inscrivent la question du génocide dans la continuité de leur lutte antérieure. Mais, comme l'a montré la sociologie des mobilisations, les cadres juridiques ne suffisent pas. Encore faut-il qu'ils soient investis et utilisés. Après la guerre civile au Liban, la reconfiguration de la communauté arménienne en France, avec l'arrivée de milliers d'Arméniens du Levant dans les années 1960, permet d'utiliser ces ressources, car elle multiplie les dynamiques et les lieux de confrontation. Si nous avons bien indiqué la mise en place d'espaces sociaux et culturels diasporiques, ceux-ci ne sont pas pour autant assez nombreux. En revanche, la vie au Levant, depuis l'arrivée des rescapés du génocide de 1915, se caractérisait par son caractère communautaire : on y parlait uniquement arménien et la communauté comprenait des médecins, des professeurs, des épiciers arméniens. Hilda Tchoboian, venue de Syrie pour vivre en France quand elle avait 17 ans, parle de ses difficultés à s'adapter à ce nouveau pays «qui était un désert culturel pour les Arméniens 53 ». Née «dans une famille Dachnak », elle décrit ainsi l'organisation de la commu-

51. Portes A., op. cit., p. 16.

52. Attarian V., «Actions pour la reconnaissance du génocide des Arméniens », in Comité de Défense de la Cause Arménienne (dir.), L'actualité du Génocide des Arméniens, Actes du Colloque à Paris-Sorbonne, Paris, Edipol, 1998, pp. 261-262.

53. Entretien avec Hilda Tchoboian, 3 mars 2015, Lyon. 
nauté arménienne en Syrie : «Quand tu es dans le parti, les enfants vont dans les écoles du parti. Il y avait les mouvements scouts, adolescents, sportifs, de bienfaisance. Tu nais Arménien, tu meurs Arménien ». Élevée dans ce contexte, une fois en France, elle s'engage très jeune pour la cause arménienne, ensuite elle devient une des responsables du parti Dachnak en Europe, et cela jusqu'en 2010. Il faut noter que le Liban de cette époque est un foyer de réflexion et de contestation, qui abrite de nombreux intellectuels et opposants ainsi que des maisons d'édition et les sièges des partis politiques. Comme le précise L. Ritter, « loin d'être un obstacle à la cohésion du groupe, ces différences leur permettent de se restituer en exil 54 ». Cette immigration génère, par exemple, le transfert d'un capital militant, dans le sens où Frédérique Matonti et Franck Poupeau l'ont conceptualisé : «incorporé sous forme de techniques, de dispositions à agir, intervenir, [...] il recouvre un ensemble de savoirs et de savoir-faire mobilisables lors des actions collectives, des luttes inter ou intra-partisanes 55 ». L'exportation de ce capital contribue à une radicalisation politique qui est façonnée par plusieurs facteurs : les fils et filles des rescapés, qui sont nés et ont grandi en France, à mi-chemin entre l'assimilation et la « réarménisation », font face au traumatisme transmis par la première génération. Dans un contexte conflictuel de la guerre froide et de forte contestation sociale, une partie d'entre eux se retrouve au sein des mobilisations de mai 68 et prend conscience du décalage entre la question du génocide des Arméniens et les revendications de l'époque. Cela est aussi lié à l'inexistence, en France, des livres sur le sujet. La reconstruction de l'espace politique arménien va mobiliser le champ universitaire et le monde de l'édition. La parution de deux premiers livres en France sur le génocide ${ }^{56}$ suscite des débats et permet d'analyser les raisons géostratégiques du génocide. Cela accélère la mobilisation en donnant un relief inédit aux revendications de justice pour lesquelles le Tribunal de Nuremberg était devenu une référence juridique. Il faut noter aussi l'effet stimulateur, sur l'espace militant arménien diasporique, de la manifestation du 24 avril 1965 qui réunit environ 150000 personnes dans les rues d'Erevan (Arménie soviétique) pour réclamer la reconnaissance du génocide et la restitution des terres arméniennes, ainsi que les mobilisations notamment en Syrie, au Liban, au Canada et aux États-Unis. Tous ces événements se complètent pour faire de la mémoire un outil mobilisateur.

Dans les récits, nous relevons plusieurs histoires du militantisme local, à Marseille, à Lyon, à Paris, à Valence ou à Saint-Etienne, qui sera la base de la lutte internationale. Manoug Pamokdjan, un Français d'origine arménienne, né, grandi à Lyon, avait 17 ans quand il a commencé à militer. Dans son récit, nous apprenons que les jeunes militants, de 1964 à 1968, demandent à tous les

54. Ritter L., La longue marche des Arméniens : histoire et devenir d'une diaspora, op. cit., p. 34.

55. Matonti F., Poupeau F., «Le capital militant. Essai de définition », Actes de la recherche en sciences sociales, $\mathrm{n}^{\circ} 155,2004$, p. 8.

56. Carzou J. M., Un génocide exemplaire, Paris, Flammarion, 1975 ; Ternon Y., Les Arméniens. Histoire d'un génocide, Paris, Seuil, 1977. 
commerçants d'origine arménienne de coller pour le 24 avril sur leurs vitrines des affiches portant la mention «Deuil national arménien » et de fermer leurs magasins pour une journée ou quelques heures. "Au début, les commerçants n'osaient pas et petit à petit ils l'ont fait », raconte-t-il 57. "Notre génération a politisé les commémorations du 24 avril » affirme Saténig Toufanian, jeune militante dans les années 1970 à Paris où elle milite toujours dans le domaine scientifique et littéraire, sans affiliation partisane ${ }^{58}$. "Ma génération ne voulait pas faire partie du monde qui pleure. C'est nous qui avons inventé la mobilisation des Arméniens en France. Nous disions : le 24 avril sera une journée de revendication, pas de deuil ou de commémoration ». Cette phrase de H. Tchoboian est répétée plusieurs fois par ceux et celles qui étaient jeunes militants dans les années 1970. «On voulait revendiquer, lutter » indique Séta Papazian, une militante de cinquante ans, fondatrice et présidente actuelle du Collectif VAN (Vigilance Arménienne contre le Négationnisme) à Paris ${ }^{59}$. La politisation se traduit aussi par la radicalisation des modes d'action et des revendications. À partir de 1975, apparaissent notamment à Paris, à Lyon et à Marseille, les premiers sit-in des Arméniens devant les consulats turcs avec des panneaux sur lesquels est écrit «La Turquie, assassin ». Comme toute manifestation sur ce sujet était interdite, nous avons recueilli plusieurs récits d'arrestations. "Au terme de plusieurs heures de garde à vue, les jeunes militants sont systématiquement conduits au Fort de Vincennes d'où ils sont libérés au milieu de la nuit » raconte un ex militant de Nor Seround qui a plusieurs fois été conduit au Fort de Vincennes à Paris. Les interdictions ne freinent pourtant pas la mobilisation : en 1976, on compte cinquante personnes à Lyon, cent à Marseille, moins à Paris. L'année suivante, il y a deux mille personnes à chaque rassemblement.

La construction politique de la mémoire qui contribue à structurer la communauté diasporique, le changement de situation des Arméniens, leurs nouvelles opportunités économiques, politiques et sociales, la configuration de la communauté avec le transfert des expériences militantes du Levant, l'air du temps politisé et les mobilisations autour de la même cause dans les différents pays se complètent pour renforcer les ressources politiques et faciliter la diffusion de la cause arménienne vers le champ politique international. Même si le parti Dachnak est l'acteur principal de cette diffusion, Ramgavar intervient aussi dans ce processus à travers la mise en place de plusieurs organisations ayant des bureaux dans les principaux pays de la diaspora : la Fédération arménienne européenne pour la Justice et la Démocratie (European Armenian Federation for Justice and Democracy-EAFJD), le Comité de défense de la cause arménienne (CDCA-1965) qui exercent un travail de lobbying pour la reconnaissance du génocide arménien au niveau international, en lien avec des

57. Entretien avec Manoug Pamokjan, 22 janvier 2015, Lyon.

58. Entretien avec Satenig Toufanian, 3 février 2015, Paris

59. Entretien avec Séta Papazian, 9 octobre 2014, Paris. 
groupes de pression équivalents dans d'autres pays. Par exemple aux ÉtatsUnis : l'Assemblée arménienne d'Amérique (Armenian Assembly of AmericaAAA-1972) et le Comité national arménien d'Amérique (Armenian National Committee-1973) 60. Cette période de réorganisation voit également le bouleversement de l'espace politique arménien en France, comme dans les autres pays, par la différenciation des acteurs et parfois par les dissensions au sein des groupes, car nombre d'organisations voient le jour avec de multiples objectifs : le Centre de recherche de documentation arménien (CRDA), le Mouvement national arménien, Solidarité Franco-Arménienne, Terre et Culture, Centre d'études arméniennes, ainsi que plusieurs journaux et des radios FM communautaires. La JAF, fondée à Marseille en 1945, autour de Hentchak et de la résistance de Manouchian, s'ouvre vers plusieurs villes de France et d'autres pays. Le Nor Seround, branche de jeunesse de Dachnak s'organise dans environ 15 pays. L’Union générale arménienne de Bienfaisance (UGAB) qui existe depuis 1906, devient la plus grande organisation à but non lucratif dans le monde, avec une présence dans 28 pays. Néanmoins, nous apprenons, par les récits, que la plupart de ces actions sont organisées par un nombre limité de personnes. S. Papazian parle d' « un travail de fourmi 61 » qui consiste, par exemple, à organiser des débats, rendre visite aux élus régionaux, municipaux, nationaux. Ce tournant idéologique, politique et structurel suscite une onde de choc et donne lieu à une extension du conflit et à une radicalisation politique. "Nous disions : Mais il n'y a rien qui change... aucune avancée » raconte $\mathrm{S}$. Toufanian ${ }^{62}$. Ce sentiment d'urgence 63 s'ajoute à l'expérience de la lutte armée forgée au Moyen-Orient, apportée par les Arméniens venus du Liban. Deux organisations : les Commandos des Justiciers du génocide des Arméniens (CJGA) et l'Armée secrète arménienne pour la libération de l'Arménie (ASALA), mènent, dans plusieurs pays, des attentats contre les représentants de l'État turc, entre 1975-1985. Une partie des militants et des chercheurs insiste sur l'effet de réveil de ces actions, une partie sur leur rôle démobilisateur. Il faut noter le durcissement des autorités des pays occidentaux vis-à-vis des manifestations pacifiques, autour des mêmes revendications que celles des activistes. Gérard Chaliand, avec la participation de trois Prix Nobel et des intellectuels de l'époque, organise en 1979 le Tribunal Permanent des Peuples, qui succède au Tribunal Russel, pour « sortir la cause arménienne du ghetto de la violence 64 ». Cet événement est le révélateur d'un nouvel espace de lutte, confirmé par F. Mitterrand, en 1984 : "Il ne doit y avoir ni malentendu ni violence entre les Arméniens et la France 65 ». Face à cet appel, les organisations arméniennes se trouvent

60. Vicken C., « Histoire, mémoire et relations internationales : la diaspora arménienne et les relations arméno-turques. ", Relations internationales, $\mathrm{n}^{\circ} 141,2010$, pp. 25-45.

61. Entretien avec Séta Papazian, 9 octobre 2014, Paris.

62. Entretien avec Satenig Toufanian, 3 février 2015, Paris

63. Minassian G., Guerre et terrorisme arméniens, 1972-1998, Paris, PUF, 2002, p. 31.

64. Chaliand G., Crime de Silence, Paris, L'Archipel, 2015, p. 8.

65. 7 janvier 1984, journal télévisé d’Antenne 2 présenté par Christine Ockrent. 
contraintes d'adoucir leurs revendications initiales et de se tourner vers le champ politique conventionnel dans lequel elles sont déjà présentes. Les Nations-Unies, par exemple, ont pris une place importante dans ce processus, quand elles ont adopté en 1948, la convention pour la prévention et la répression du crime de génocide. Nous y observons ce qu'Isabelle Sommier nomme un «pragmatisme radical 66 ", associant leurs buts radicaux et les solutions immédiates, les actions et le lobbying. Suite aux travaux «dans les couloirs, dans la préparation des travaux des commissions » des militants de CDCA, la première réussite ne tarde pas : dans le rapport de la Commission des Droits de l'Homme de l'ONU (1979), le paragraphe 30 sur la prévention des génocides mentionne le génocide de 1915. Si après l'éclatement d'une crise diplomatique avec la Turquie, la Commission supprime ce paragraphe, il sera adopté en 1985. Il faut noter l'adoption d'une résolution similaire, en 1987, du Parlement européen, et la déclaration en 1998 de l'Assemblée parlementaire du Conseil de l'Europe. «L'État turc a cru que nous étions très organisés, avec des relations en haut. Mais ce n'était pas vrai » suppose M. Amtablian 67. Nous pouvons maintenant voir les ressources de cette apparence. Même si «les enjeux diplomatiques et stratégiques contraignent fortement les comportements étatiques 68 », « le travail de fourmi » a un impact social qui influence les champs politiques, en renforçant des opportunités de manière continue. En s'appuyant sur l'opinion publique des pays d'accueil, par exemple de la France, les entrepreneurs de la cause arménienne, unis depuis 1994 autour du Comité de coordination des Arméniens de France (CCAF), gagnent les institutions internationales, comme le dit $\mathrm{H}$. Tchoboian : «Ce qu'on a réussi en France, c'est d'avoir l'opinion publique à nos côtés. C'est grâce à cela que le génocide de 1915 a été reconnu par le champ politique 69 ».

À partir de cette période, les communiqués des organisations arméniennes en France, ainsi que d'autres pays sauf l'Arménie et la Turquie, parlent au nom de la diaspora. Nous avons maintenant plusieurs éléments pour voir le rôle des organisations politiques dans la construction de la diaspora et sa cristallisation. Rappelons notre question initiale : pourquoi cette auto-désignation émerge-t-elle dans les années 1970 ? Il faut noter au préalable que la diaspora arménienne n'a pas de représentativité sur la scène politique internationale qui reconnait uniquement les représentants des populations vivant en Arménie et en Turquie, même si dans ces territoires la proportion de population est beaucoup moins importante par rapport à la diaspora. Les enjeux d'auto-désignation gagnent de l'importance surtout à partir de la construction de la revendi-

66. Sommier I., Le renouveau des mouvements contestataires à l'beure de la mondialisation, Paris, Flammarion, 2003.

67. Entretien avec Mihran Amtablian, 29 janvier 2015, Lyon.

68. Dequierez G., «Les formes du discours victimaire et leur réception politique. Le cas de militants tamouls sri lankais en France ", in Lefranc S., Mathieu L. (dir), Mobilisations de victimes, Rennes, PUR, 2009, p. 120.

69. Entretien avec Hilda Tchoboian, 3 mars 2015, Lyon. 
cation du statut de victime et de la reconnaissance du génocide qui nécessite ce statut diasporique car cette revendication ne peut pas être portée par les Arméniens de Turquie et d'Arménie où il est difficile, à l'époque, de s'organiser autour de cette cause. L'exemple de la Turquie nous montrera comment les contestations en Turquie et en diaspora débouchent sur des rapports très différents à la répression politique.

\section{Renaissance de l'activisme arménien en Turquie}

De nombreux travaux soulignent combien la répression peut décapiter un mouvement et freiner la mobilisation ${ }^{70}$. C'est le cas du mouvement arménien en Turquie qui a attendu, pour renaître, jusqu'à la fin des années 1990. Il faut noter que dans ce contexte, la construction de la nation turque autour d'une identité musulmane a conduit non seulement au génocide arménien mais également à plusieurs déplacements de populations non-musulmanes au cours du $\mathrm{XX}^{\mathrm{e}}$ siècle. Les Arméniens demeurés en Turquie restent invisibles, marginaux et sans voix, jusqu'en $1996{ }^{71}$, car ils sont définis comme "Infidèles » ou «ennemis intérieur » par les représentants de l'État. Cette phrase d'un journaliste arménien est emblématique : «Parfois, quand je dis que je suis Arménien, je me demande si, par hasard, je ne suis pas en train de m'insulter $72 »$. Dans ce contexte, le Patriarche (patrikhane) qui fait figure, comme à l'époque ottomane, de façade institutionnelle de la communauté, prend de la distance vis-àvis de la diaspora. Suite au coup d'État militaire de 1980, l'hostilité fanatique anti-arménienne se renforce davantage. Pourtant, à la fin des années 1980, en dépit du climat de guerre qui institutionnalise la structure militariste du champ politique en Turquie, on assiste à une évolution de la reconnaissance de la question kurde et à l'émergence de nouveaux mouvements contestataires autour de causes inédites auparavant, comme le mouvement féministe ou LGBT. Ces derniers se renforcent en profitant des fenêtres d'opportunité que les institutions européennes leur fournissent, à partir de 1987, date de la candidature de la Turquie à l'UE (à l'époque, Communauté européenne). Elle sera officiellement reconnue candidate en 1999. Il ne s'agit pas, à proprement parler, d'un contexte idéal pour l'émergence d'un mouvement contestataire autour de la question arménienne, mais d'une ouverture qui fournit aux intellectuels arméniens un espace de travail sur les questions culturelles. Ce travail ne se transforme pas en action mais met en place des cercles autour de la question. La reconnaissance des revendications des mouvements arméniens de la diaspora, donc la visibilité de la question arménienne dans les pays occidentaux et l'indépendance définitive de la République d'Arménie, en 1991, convergent pour renforcer ce climat. Les publications de plusieurs livres sur la

70. Olivier J., «State Repression and Collective Action in South Africa 1970-84 », South African Journal of Sociology, $\mathrm{n}^{\circ} 22,1991$, pp. 109-117.

71. Karakaşlı K., Kentel F., Özdoğal G., Üstel F., op. cit., p. 25.

72. Sarkis Saropyan, Agos, 7 juin 2015. 
culture et sur l'histoire arméniennes ${ }^{73}$, la création de la maison d'édition Aras (1993) déclenchent un processus de réflexion. Mais cela va de pair avec un renforcement de l'hostilité anti-arménienne. L'État turc, d'un côté tente de donner à la communauté internationale une image de démocratie en laissant place aux travaux culturels, d'un autre côté, il accentue le discours sur des «ennemis arméniens 74 ». "C C'était un temps très dur. Dans la presse nous suivions les "prétendues relations entre le PKK et les Arméniens". Nous savions que c'était des mensonges mais nous ne pouvions pas nous exprimer ${ }^{75}$ ».

L'apparition du journal Agos (sillon en arménien) ouvre, en 1996, la voie à la contestation légale pour la cause arménienne en Turquie. «Il fallait faire quelque chose » nous dit Karin Karakaşli, une des responsables du journal. Pour elle, l'expression «sortir de l'impasse » est une clé de la politique d'Agos : « dans le pays, tout était contre nous. Tout nous obligeait à nous taire ou bien à nous révolter de façon réactionnaire. Au milieu d'une telle hostilité, c'était comme un rêve de parler, de vivre ensemble 76 ». Bilingue (turco-arménien), l'hebdomadaire définit son but politique : "Vivre ensemble en évitant de se ranger dans tel ou tel camp 77 ». Elle entre dans le cas de figure décrit par Erik Neveu lorsqu'il écrit : "les mouvements peuvent se trouver devant la contrainte de "traduire" leurs revendications et actions dans des formats correspondant aux temps et aux exigences de simplification, de force visuelle des médias 78 ». La remise en cause des fondements de la République turque commence, dans cette optique, avec la question rendue publique par Agos : «Où sommes-nous ? " qui fait référence aux Arméniens déguisés sous une identité musulmane pendent le génocide de 1915. Bien qu'elle mette entre parenthèses le terme génocide, cette question entame la déconstruction de l'identité nationale dominante et propose une autre version de l'histoire, qui subvertit l'histoire officielle. Les nombreuses publications et actions autour de cette question font prendre conscience à de nombreux Turcs de l'existence de la communauté arménienne, dont les membres sont restés confinés entre eux depuis le génocide. Novateur, le mouvement social évoluant autour d'Agos déclenche un processus qui n’a été jamais tenté ni exprimé auparavant en Turquie.

Cependant le but politique exprimé par Agos va à l'encontre des revendications de la diaspora arménienne dans laquelle les Arméniens vivant en Turquie sont vus comme les « otages de l'État turc ». Ce regard n'a pas changé après l'émergence de l'Agos, car cette fois-ci apparait l'idée de la récupération

73. Il faut parallèlement signaler les éditions Belge, qui publient par exemple les œuvres d'Y. Ternon ou de V. Dadrian.

74. Danzikyan Y., «12 eylül ve 11 Ermeni » («12 septembre et 11 Arméniens »), Radikal, 2 avril 2012.

75. «Interview avec Hrant Dink », Cumburiyet, 5 avril 1996.

76. Entretien avec Karin Karakaşl1, 16 mars 1995, par skype.

77. Dink H., «Une seule voie : vivre ensemble », Agos, 16 juin 2006, traduit par François Skvor.

78. Neveu E., op. cit., p. 508. 
du mouvement arménien par l'État turc. Selon Tigrane Yegavian par exemple, la Turquie, en s'appuyant sur ce mouvement, réussit à faire passer le militantisme de la diaspora arménienne pour de l'extrémisme ${ }^{79}$. "À partir du moment où on demande la justice, on est perçu comme des extrémistes » insiste S. Papazian. "Chaque fois qu'on réussissait quelque chose au Parlement européen, la presse turque diabolisait la diaspora comme des extrémistes. Et Hrant Dink est entré dans ce jeu en stigmatisant la diaspora, en parlant de l'extrémisme de la diaspora » affirme M. Amtablian. Les membres de CDCA de l'époque disent que l'État turc a utilisé ce conflit. Ils expliquent ainsi l'oubli du Parlement européen de son propre appel, fait le 28 septembre 2005, à la Turquie, de reconnaître le génocide des Arméniens, considérant « cet acte comme un préalable à l'adhésion à l'Union européenne ». Cet appel ne va pas être répété et la reconnaissance du génocide ne sera plus une condition d'adhésion pour la Turquie. «Hrant Dink était invité au Parlement européen pour dire qu'il travaillait pour la démocratisation de la Turquie, que la diaspora ne peut pas se mêler à cette question. Il insistait sur la légitimité des Arméniens de la Turquie pour parler de cette question parce qu'ils sont des citoyens de la Turquie. Hrant Dink était pour l'entrée de la Turquie sans condition et il revendiquait que la question arménienne est une question interne à la Turquie. Et cela convenait à l'État turc et aux États européens » raconte $\mathrm{H}$. Tchoboian ${ }^{80}$. Nous voyons que l'auto-désignation de la diaspora arménienne est liée aux ruptures politiques entre les deux espaces de contestation. Cette rupture est bien visible dans l'article de H. Dink, intitulé «Faire connaissance avec l'Arménie ", qui invite les Arméniens à « se tourner maintenant vers le sang neuf de l'Arménie indépendante, seule capable de les libérer du poids de la diaspora et rejeter ainsi symboliquement la part altérée de leur sang turc». Repris par la presse de la droite radicale qui en publie des citations apocryphes, cet article fait condamner son auteur, le 12 juillet 2006, à six mois de prison pour «insulte à l'identité turque 81 ». Surtout, quand il écrit qu'une des filles adoptives d'Atatürk pouvait être issue d'un orphelinat arménien ${ }^{82}$, la déclaration du Secrétariat général de l'Armée montre l'inflexibilité de la structure étatique : " ouvrir le débat sur un tel symbole est un crime contre l'intégrité nationale et la paix sociale 83 ». Dans sa dernière chronique, il décrit sa situation avec une référence au génocide de 1915 : «Certaines personnes ont décidé que désormais ce Hrant commençait à être de trop et qu'il convenait de lui fixer des limites... tout comme le firent nos ancêtres en $1915^{84}$ ». L'article se termine par son refus de se mettre en route comme à

79. Yegavian T., "Entre rêve et réalisme : pour un nouveau dialogue arméno-turc ", Repairfuture, 2014, URL : http://www.repairfuture.net/index.php/fr/pardon-reconciliationdialogue-point-de-vue-de-la-diaspora-armenienne/entre-reve-et-realisme-pour-un-nouveau-dialogue-armeno-turc (consulté le 5 février 2015).

80. Entretien avec Hilda Tchoboian, 3 mars 2015, Lyon.

81. http://fr.rsf.org/turquie-le-journaliste-armenien-hrant-dink-19-07-2006,14388.html

82. Dink H., «Le secret de Madame Sabiha », Agos, 4 avril 2006.

83. Dink H., « Pourquoi ai-je été pris pour cible ? ", Agos, 17 janvier 2007, traduit par François Skvor. 
l'époque du génocide. «Par conséquent nous resterons et nous lutterons ${ }^{85}$ ».

Le 19 janvier 2007, Hrant Dink est abattu de trois balles dans la tête. Sa mort déclenche une vague de protestations et de solidarités. Doug McAdam, Sidney Tarrow et Charles Tilly parlent du changement d'échelle, pour désigner un « changement dans le nombre et le niveau des actions protestataires coordonnées qui conduisent à une protestation élargie regroupant un spectre plus large d'acteurs $86 »$. Cette définition nous autorise à considérer cet assassinat comme un changement d'échelle, parce qu'autour d'Agos, elle fait cristalliser de manière précipitée, un nouveau mouvement social : malgré ses disparités, il réalise l'union de différents groupes « agissant avec une certaine continuité pour promouvoir un changement dans la société 87 ». Les activités, d'abord circonscrites aux milieux intellectuels, touchent un public plus important et les bases sociales de recrutement du mouvement s'élargissent. Plusieurs associations, initiatives, collectifs de la cause arménienne apparaissent, comme l'organisation de la jeunesse arménienne Nor Zartonk (le nouveau réveil) qui a un discours plus radical. Par ses actions, son journal et sa radio, cette organisation autogérée joue un rôle de réseau d'information de l'espace militant multi-organisationnel en Turquie. Son approche, qui accentue l'intersectionnalité, lui permet d'élargir son réseau militant. À partir des années 2010, nous assistons à l'émergence de nouveaux modes de mobilisation et de manières de penser les revendications relatives au génocide de 1915 : le terme génocide est devenu un objet de la revendication. La mutation visible des formes de ses revendications, transforme progressivement les relations de ce mouvement avec la diaspora.

\section{Les réseaux emportent les structures}

À partir de ces deux cas, nous comprenons que les entrepreneurs de la cause arménienne, en développant des méthodes dissemblables d'adaptation tactique face aux différentes difficultés propres à leurs territoires de contestation, contribuent à co-construire ces contextes. Tout en assemblant et investissant leurs ressources avec des méthodes parfois contradictoires, ils apprennent à adapter leurs objectifs à ces ressources qui se modifient dans un processus complexe et dynamique. «Les travaux de fourmi » menés dans deux espaces détachés, ont un impact social qui influence le champ politique international : actuellement ce crime est reconnu officiellement comme " génocide » dans vingt-trois pays et dans toutes les institutions politiques internationales. En outre, dans la plupart des grandes villes européennes, ainsi qu'étatsuniennes et canadiennes, ont été érigés des monuments à la mémoire

84. Ibid.

85. Ibid.

86. McAdam D., Tarrow S., Tilly C., Dynamics of contention, Cambridge, Cambridge University Press, 2001, p. 387.

87. Turner R., Killian L., Collective Behavior, Englewood Cliffs, Prentice Hall, 1972, p. 246. 
des victimes de 1915. Nous observons également une prise en considération de cette question dans les domaines de la recherche, de l'éducation, des publications, des créations artistiques. Cependant une partie des jeunes militants de la diaspora parle de l'enlisement du processus de la reconnaissance en insistant sur le fait que «cette demi-victoire démobilise le mouvement ». G. Chaliand, quant à lui, durant les grands événements du centenaire du génocide en 2015, nous a dressé ce constat : "De zéro, avec des moyens dérisoires, on a atteint des résultats importants. À partir de maintenant le militantisme des Arméniens va baisser. On ne pourra pas avoir la même visibilité au 101e anniversaire 88 ». En effet, en 2016, les commémorations de 101e anniversaire avaient une faible visibilité. Pourtant, les effets de ces mobilisations qui se propagent dans d'autres espaces sont assez complexes pour cette prévision rapide.

L'internationalisation de cette question à partir de 1980 et jusqu'à nos jours, a multiplié les ressources des mobilisations arméniennes en diaspora ainsi qu'en Turquie. Mais en même temps, comme Vicken Cheterian le précise, « l'émergence de l'Arménie indépendante en 1991 fit basculer le centre de gravité de la politique arménienne des communautés de Beyrouth, Paris ou Los Angeles à Erevan ${ }^{89}$ ». Quant à Laurence Ritter, qui vit actuellement à Erevan, elle nous explique que ce basculement ne remonte pas à 1991 : «Erevan est depuis au maximum une dizaine d'années en train de s’imposer comme un point de rencontre de la multitude des diasporas. Significativement, nous avons même un ministère de la Diaspora, mais tout ceci est récent 90 ». En tout cas, nous observons que les organisations de la diaspora accordèrent à l'Arménie un soutien politique, en créant un espace pour une politique proarménienne dans les principales capitales occidentales. "Aller en Arménie pour soutenir le pays» devient désormais une tendance forte dans la diaspora occidentale, notamment en France. Ces voyages suscitent des liens commerciaux qui traversent les frontières en créant un territoire circulatoire ${ }^{91}$ et un espace social transnational.

Par ailleurs, depuis le début des années 2000, un troisième espace militant arménien s'ajoute à ceux de la diaspora et de la Turquie : un nouveau mouvement contestataire provoque, en Arménie, plusieurs innovations organisationnelles, idéologiques et de modes d'action. Composé de multiples groupes, associations, ce mouvement conteste à la fois les gouvernements arménien et turc à partir de nouvelles sources d'influence. Ces innovations sont en train de s'élargir aux autres secteurs de la vie sociale grâce aux réseaux qui se mettent en place. Cela peut nous autoriser à utiliser le concept de cycle de mobilisa-

88. Entretien avec Gérard Chaliand, 5 octobre 2015, Nice.

89. Cheterian V., "Histoire, mémoire et relations internationales : la diaspora arménienne et les relations arméno-turques. ", Relations internationales, $\mathrm{n}^{\circ} 141,2010$, pp. 25-45.

90. Entretien avec Laurence Ritter, 2 mai 2016, par internet.

91. Tarrius A., Costa-Lascoux J., Hily M. A., «Au-delà des États-nations : des sociétés de migrants ", Revue européenne des migrations internationales, vol. 17, n 2, 2001, pp. 37-61. 
tion 92 proposé par $\mathrm{S}$. Tarrow, au sens plus large et conforme à cet espace en mutation. Ce cycle suscite de nouvelles alliances, avec les militants arméniens de la diaspora et avec les mouvements arméniens, féministes, antimilitaristes, LGBT de Turquie. Les militants de Pink Armenia, organisation LGBT d'Arménie, par exemple, participent à des réunions avec les autres organisations féministes, queer ou LGBT en Turquie. Entre les groupes féministes de deux pays il y a aussi plusieurs projets communs et rencontres, en lien avec le Nor Zartonk.

À partir de la diaspora arménienne, en France, nous avions expliqué comment les organisations politiques créent des ponts qui suscitent l'émergence d'un champ social multipolaire, étendu sur plusieurs territoires, traversés par le réseau militant. Dans un premier temps, les clivages politiques freinent l'élargissement de ce réseau, surtout vers l'Arménie et la Turquie. Pourtant, en dépit de la différence des opportunités et priorités, l'interdépendance de ces mouvements par le fait de lutter autour de la même question et de s'appuyer sur le même événement historique, suscite une interaction continue. Depuis 2010, nous pouvons témoigner d'un rapprochement entre ces trois espaces militants. Comme les revendications autour de la réparation font désormais irruption dans le mouvement arménien en Turquie, plusieurs initiatives, en relation avec la diaspora et l'Arménie, apparaissent autour de la demande de justice. Par exemple, le Collectif 2015 Réparations, à l'initiative de l'Association Terre et Culture, demande au gouvernement turc, la restitution des biens arméniens à usage collectif confisqués par l'État. Nombre de militants vivant en Turquie participent à cette campagne portée par la diaspora. La différence de participation ne suscite pas, cette fois-ci, de ruptures : «Ils font ce qu'ils peuvent » affirme une militante parisienne de cette association.

Quant aux structures traditionnelles, elles perdent leur rôle principal dans la reconstruction du mouvement. Yériche Gorizian, jeune universitaire en droit, ex-dirigeant de Nor Seround, branche jeunesse de Dachnak, ayant actuellement une position critique vis-à-vis des organisations traditionnelles, indique qu’à partir des années 1980, celles-ci « ont majoritairement mis en suspens la question de la justice et ont limité leurs revendications à la seule reconnaissance du génocide, davantage par souci tactique que par volonté idéologique. Mais aujourd'hui cette tactique est devenue un réflexe du discours militant actuel qui s'est surtout installé du fait d'un manque de réflexions de fond sur l'avenir de la cause 93 ». Plusieurs militants, âgés de 19 à 25 ans, insistent sur le fait que les structures traditionnelles, intégrées au champ politique conventionnel, freinent la mobilisation. Ce constat se retrouve dans cet extrait d'entretien avec un jeune militant qui préfère conserver l'anonymat : « Il faut

92. Tarrow S., Democracy and disorder: Protest and politics in Italy, 1965-1975, Oxford, Oxford University Press, 1989.

93. Entretien avec Yeriche Gorizian, 19 novembre 2015, Lyon. 
abolir les vieilles structures et les remplacer par les nouvelles. Les vieux militants ont donné ce qu'ils pouvaient donner. De toute façon le changement de génération va se faire. La grande question c'est de savoir si la jeunesse est en mesure de créer des organisations qui vont pouvoir déclencher une dynamique nouvelle et les convergences probables entre les espaces ${ }^{94} »$. Avec les mêmes critiques, plusieurs militants de Nor Seround ont quitté leur organisation. Leur décision de créer le Comité de soutien français à Nor Zartonk et agir au nom de ce comité autogéré est un exemple du rapprochement entre les deux espaces. La mobilisation se reconstruit en adoptant des formes de campagnes communes, en particulier sur les microprojets de réparation relatives au génocide de 1915. Dans le contexte où «l'inégalité des moyens étatiques et financiers est criante 95 », les militants associent, comme l'avaient fait leurs antécédents, leurs buts radicaux aux opportunités présentes.

«Depuis l'indépendance de l'Arménie et la poussée de la mondialisation, les Arméniens de la diaspora n'ont plus besoin des réseaux communautaires traditionnels pour exprimer leur identité arménienne », souligne Gaïdz Minassian ${ }^{96}$. Si la mondialisation du capital a renforcé les frontières politiques et sociales pour les populations, il est fort apparent que les distances se raccourcissent grâce aux nouvelles technologies. C'est aussi le constat de L. Ritter pour qui, aujourd'hui, l'indépendance de l'Arménie et la circulation de l'information grâce au numérique a profondément bouleversé le fonctionnement de la diaspora traditionnelle ${ }^{97}$. Selon elle, cette mise en réseau n'est pas seulement limitée à un cybermonde virtuel; elle permet aussi de véritables liens, culturels notamment, d'informer, de faire circuler les informations. Les relations sociales et politiques qui lient les militants à différents lieux géographiques se renforcent également par la rapidité de déplacement. Ces multiples liens donnent lieu à des interactions mutuelles qui font naître des croisements des réseaux militants transversaux, autour de nouveaux modes de mobilisation, de nouvelles actions transnationales initiées par les militants non-institutionnels de différents pays. Cela permet une interactivité inédite et plus rapide.

Nous observons la naissance d'un nouveau registre militant qui n'appartient pas aux clivages traditionnels territoriaux et/ou politiques, ainsi qu'un nouveau réseau militant fondé sur un espace transnational lui permettant l'accès à de nouvelles ressources. L'autonomisation du réseau militant vis-à-vis des structures politiques traditionnelles favorise l'intermédiation ${ }^{98}$ entre les

94. Entretien avec L.A, 12 décembre 2015, Paris.

95. Kalfayan R., « 2015 : cap sur les réparations du Génocide arménien », Nouvelles d'Arménie, 19 décembre 2014.

96. Minassian G., "Les évolutions dans les revendications de la reconnaissance du génocide des Arméniens ", actes non-publiés du colloque Regards croisés cent ans après le génocide des arméniens : mobilisations, revendications, justice, ENS de Lyon, 6 mai 2015.

97. Ritter L., La longue marche des Arméniens : histoire et devenir d'une diaspora, op. cit., p. 279.

98. Tilly C., Tarrow S., Politique(s) du conflit, Paris, Presses de Sciences Po, 2008, p. 63. 
espaces militants arméniens multilatéraux, la fluidité facilite la diffusion des concepts, des savoir-faire, des opportunités, notamment des revendications autour de la justice, dans une logique de convergences. L'analyse d'Ariel Colonomos sur les réseaux informels dans les espaces militants est valable aussi pour notre objet de recherche : «dans cette informalité se compose une pluralité de liens qui soudent temporairement les membres d'un groupe les uns aux autres en les insérant dans une série d'échanges susceptibles d'évoluer rapidement 99 ». Temma Kaplan propose l'expression de communautés de mouvement social pour désigner les réseaux d'activistes organisés de manière informelle ${ }^{100}$. Les mouvements autour de la cause arménienne se reconstruisent en englobant les formes d'organisation fluides ou passagères, les communautés militantes et les réseaux sociaux qui s'appuient sur les structures préexistantes, sur les collectifs éphémères et sur les dynamiques complexes de ces espaces. Ils se densifient et se diversifient, sans se centraliser mais en englobant une multiplicité de groupes et d'individus, dont la plupart appartiennent à la nouvelle génération.

La configuration des interactions militantes transnationales reste encore à étudier parce qu'elle est récente et d'autant plus complexe qu'elle obéit parfois aux particularités des pays et des milieux, ainsi qu'à la configuration de l'espace politique international. Notre recherche vise principalement à montrer que pour comprendre ce qui est nouveau, il nous faut l'aborder à partir des transformations sur lesquelles il s'appuie. Revisiter ces transformations permet de remettre en question les conceptions réductrices de l'action collective et montre que les facteurs macrosociologiques sont insuffisants pour expliquer la contestation. Elle met en lumière la complexité des dimensions de l'action contestataire, en éclairant à la fois l'influence de l'environnement sur l'action collective et le caractère non structurel, relationnel, dynamique et par conséquent complexe de celle-ci.

99. Colonomos A., «Sociologie et science politique : réseaux, théories et objets d'études ", Revue française de science politique, vol. $45, \mathrm{n}^{\circ} 1,1995$, p. 32.

100. Kaplan T., Taking back the streets, Berkeley, University of California Press, 2004. 